\title{
Short-Term Correlation of the Psoriasis Area Severity Index, the Nail Psoriasis Area Severity Index, and the Dermatology Life Quality Index, before and after Treatment, in Patients with Skin and Nail Psoriasis
}

\author{
Christos Prevezas $^{\mathrm{a}} \quad$ Alexander C. Katoulis $^{\mathrm{a}}$ Evangelia Papadavid $^{\mathrm{a}}$ \\ Pantelis Panagakis ${ }^{b}$ Dimitrios Rigopoulos ${ }^{b}$ \\ a Second Department of Dermatology and Venereology, Attikon University General Hospital, Medical School, \\ National and Kapodistrian University of Athens, Athens, Greece; ${ }^{b}$ First Department of Dermatology and \\ Venereology, Andreas Syggros Hospital for Skin and Venereal Diseases, Medical School, National and \\ Kapodistrian University of Athens, Athens, Greece
}

\section{Keywords}

Skin · Nail · Chronic plaque psoriasis · Nail psoriasis ·

Clinical severity index $\cdot$ Quality of life

\begin{abstract}
Background: The response to treatment in patients suffering from skin and nail psoriasis is quantitatively evaluated by monitoring clinical severity and quality of life indexes, especially in the context of clinical trials. Among them, the psoriasis area severity index (PASI), the nail psoriasis area severity index (NAPSI), and the dermatology life quality index (DLQI) are currently the most widely used indexes. Objective: To study the short-term correlation between PASI, NAP$\mathrm{SI}$, and DLQI in patients with skin and nail psoriasis at baseline and 3 months after treatment initiation. Materials and Methods: This is a 2-center prospective, observational, crosssectional study of 40 treatment-naive patients suffering from chronic plaque psoriasis with nail involvement and requiring systemic therapy. Disease activity and quality of life were assessed at baseline and 3 months after treatment initiation by measuring PASI, NAPSI, and DLQI scores. Statistical correlations between theses indexes were investigated using the appropriate statistical tests. Results: A positive,
\end{abstract}

(c) 2019 S. Karger AG, Basel

E-Mail karger@karger.com

www.karger.com/sad strong correlation was observed between DLQI and NAPSI at baseline $(r=0.579, p<0.001)$ and at 3 months $(r=0.484$, $p=0.002)$. Similarly, a strong correlation was noted between DLQI and PASI at baseline $(r=0.496, p=0.001)$ and at 3 months ( $r=0.401, p=0.01)$. Moreover, we observed a positive strong correlation ( $r=0.515, p=0.001)$ between DLQI at baseline and NAPSI at 3 months and a moderate correlation ( $r=0.433, p=0.005)$ between DLQI at baseline and PASI at 3 months. Conclusion: The observed positive correlations between PASI, NAPSI, and DLQI provide further evidence in support of their simultaneous utilization in clinical studies for skin and nail psoriasis. Moreover, the predictive value of DLQI at baseline emphasizes the importance of quality of life assessment when designing a therapeutic study as well as in the everyday clinical practice.

(c) 2019 S. Karger AG, Basel

\section{Introduction}

Psoriasis is a chronic inflammatory skin disease that often affects the nails, significantly impairing the healthrelated quality of life. Several quantitative tools have been proposed in order to evaluate the clinical severity, the psy- 
Fig. 1. Flow chart of the study patients.

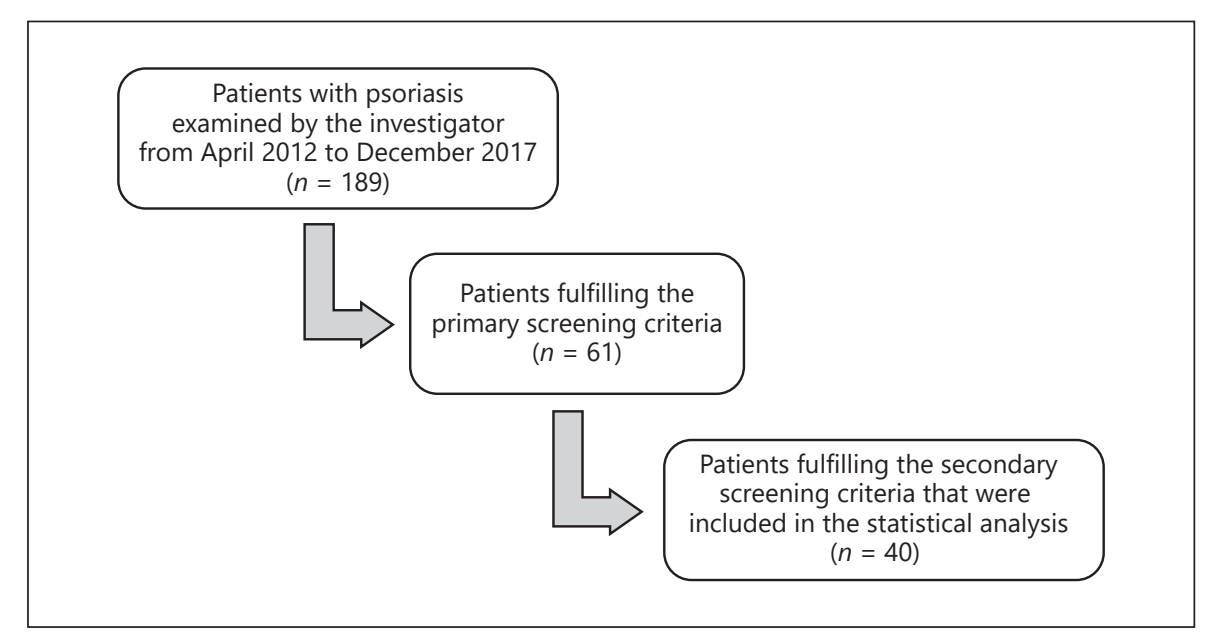

chological burden, and the response to treatment [1]. Among these tools, the psoriasis area severity index (PASI) and the nail psoriasis area severity index (NAPSI) are physician-related indexes that have been widely used in clinical trials in order to evaluate the severity of skin and nail diseases, respectively $[2,3]$. Moreover, the quality of life before and after treatment is usually assessed with the dermatology life quality index (DLQI), a patient-related tool with a high degree of reliability and reproducibility [4].

Nail involvement is common in psoriasis. The prevalence of nail disease among psoriatic patients is reported to be up to $56 \%$, whereas, the lifetime incidence reaches $80 \%$ [5]. This association should not be overlooked as emerging therapies are claiming to attain a more rapid and efficient clinical response in certain disease phenotypes, including nail psoriasis. More importantly, nail psoriasis has a strong and independent impact on the patient's quality of life [6].

In the present study, we sought to investigate the shortterm correlation between PASI, NAPSI, and DLQI and at different time points with respect to treatment in patients with skin and nail psoriasis. Our further aim was to assess their possible predictive value and to evaluate their simultaneous utilization in clinical studies.

\section{Materials and Methods}

This is a 2-center, prospective, observational, cross-sectional study. Patients were recruited from the psoriasis clinics of 2 university hospitals, both located in Athens, Greece. The observational period lasted from April 2012 until December 2017.

An initial screening was performed based on the following inclusion criteria: (1) treatment-naive patients; (2) clinical diagnosis of chronic plaque psoriasis; (3) coexistence of nail involvement; (4)
Table 1. Demographic characteristics of the study population

\begin{tabular}{lc}
\hline Characteristic & Value \\
\hline Age, years & $49.63 \pm 13.73$ \\
$\quad$ Range & $25-79$ \\
Sex & \\
$\quad$ Male & $24(60)$ \\
Female & $16(40)$ \\
Education & \\
Elementary school & $2(5)$ \\
High school & $19(47.5)$ \\
University & $19(47.5)$ \\
Profession & $3(7.5)$ \\
Unemployed & $6(15)$ \\
Freelancer & $5(12.5)$ \\
Housewife & $8(20)$ \\
Retired & $18(45)$ \\
Employee & \\
Race & $40(100)$ \\
Caucasian & \\
Residence & $40(100)$ \\
Athens, Greece &
\end{tabular}

Values are presented as means \pm SD or numbers (\%) unless otherwise stated. The total number of patients was 40 .

moderate to severe disease requiring systemic therapy, i.e., PASI $\geq 10$ and $10<$ DLQI $\geq 10$ or PASI $<10$ and DLQI $\geq 10$, and nail disease of any extent; (5) absence of arthritis or other psoriasis comorbidities that may affect the quality of life. Patients who fulfilled the inclusion criteria were enrolled into this study.

A total of 61 patients were included in this study. Patients were assessed at baseline, and 3 months after treatment initiation by a single investigator (C.P.). The following indexes were recorded during each visit: PASI (range 0-72), NAPSI (range 0-160; evaluating all nails), and DLQI (range $0-30$ ). Only data from patients who responded to treatment $(n=40)$ were included in the statisti- 
Table 2. Longitudinal index analysis (parametric and nonparametric)

\begin{tabular}{lcccccc}
\hline & \multicolumn{2}{l}{ Parametric } & & & Nonparametric & \multirow{2}{*}{$p$ value } \\
\cline { 2 - 3 } & baseline & 3 months & & baseline & 3 months & \\
\hline PASI & $9.7 \pm 5.09$ & $2.6 \pm 1.86$ & & $10.55(7.3)$ & $2.3(3.5)$ & $<0.001$ \\
DLQI & $16.58 \pm 4.72$ & $3.75 \pm 4.04$ & & $14.5(9)$ & $3(3)$ & $<0.001$ \\
NAPSI & $33.4 \pm 23.44$ & $17.8 \pm 15.16$ & & $25(30.8)$ & $15(19)$ & $<0.001$ \\
\hline
\end{tabular}

Values are presented as means \pm SD or medians (IQR).
Table 3. Statistical correlation of indexes at baseline and at 3 months

\begin{tabular}{|c|c|c|c|c|c|c|c|c|}
\hline & \multicolumn{4}{|c|}{ Baseline } & \multicolumn{4}{|c|}{3 months } \\
\hline & DLQI & $p$ & NAPSI & $p$ & DLQI & $p$ & NAPSI & $p$ \\
\hline \multicolumn{9}{|l|}{ Pearson's $r$} \\
\hline PASI & 0.496 & 0.001 & 0.211 & 0.192 & 0.401 & 0.01 & 0.196 & 0.224 \\
\hline DLQI & - & & 0.579 & $<0.001$ & - & & 0.484 & 0.002 \\
\hline \multicolumn{9}{|l|}{ Spearman's $r$} \\
\hline PASI & 0.543 & $<0.001$ & 0.288 & 0.072 & 0.574 & $<0.001$ & 0.215 & 0.102 \\
\hline DLQI & - & & 0.5 & 0.001 & - & & 0.357 & 0.024 \\
\hline
\end{tabular}

cal analysis (secondary screening). Response to treatment was defined as a decrease of any level in all 3 indexes at the 3-month follow-up visit. The patient flow charts and the demographic characteristics of the study population are presented in Figure 1 and Table 1, respectively.

\section{Therapeutics}

Only evidence-based systemic treatments for psoriasis were used. The distribution by therapeutic regimen was as follows: 9 patients received oral methotrexate at a fixed single weekly dose ranging from 7.5 to $12.5 \mathrm{mg}$ plus $5 \mathrm{mg}$ of folic acid the day after methotrexate intake; 20 patients received oral apremilast $(30 \mathrm{mg}$ b.i.d.) following an initial titration according to the drug's protocol; 9 patients received subcutaneous ustekinumab (45 $\mathrm{mg}$ ) at week 0 and week $4 ; 1$ patient received subcutaneous secukinumab (300 mg) at weeks $0,1,2$, and 3 , followed by monthly maintenance dosing starting at week $4 ; 1$ patient received oral ciclosporin at a stable daily dose of $3 \mathrm{mg} / \mathrm{kg}$. No one received combination therapy either with a topical or another systemic agent.

\section{Statistical Analysis}

All statistical tests were two sided and performed with SPSS version 17.00 (SPSS Inc., Chicago, IL, USA). The distribution of the variables was assessed using the Kolmogorov-Smirnov test and a normal probability plot. A paired samples $t$ test was selected for comparison of indexes at 2 different time points (baseline and 3 months). A Wilcoxon test was used when the distribution of the variables was not normal. Correlations among indexes were evaluated using Pearson's correlation coefficient and Spearman's rank correlation coefficient. The level of statistical significance was set at $5 \%(p<0.05)$. Borderline statistically significant values $(0.05<$ $p<0.1)$ were also recorded.

\section{Results}

The parametric and nonparametric longitudinal index analysis is summarized in Table 2 . They both depict a statistically significant $(p<0.001)$ decrease in all 3 indexes 3 months after treatment initiation. This finding suggests that these indexes were able to detect changes due to treatment, corroborating our further statistical exploration.

The statistical correlations between indexes at baseline and 3 months are summarized in Table 3. A positive, strong correlation was observed between DLQI and PASI, as well as between DLQI and NAPSI, at both time points. The strongest correlation was found for DLQI and NAPSI at baseline ( $r=0.579, p<0.001$, parametric analysis). On the other hand, a weak-to-moderate correlation was found between PASI and NAPSI at both times points.

To investigate the before-/after-treatment correlation, and to test the hypothesis of using a baseline index as a predictive tool for treatment response, we performed the same analysis, this time comparing the baseline with the 3 -month values (Table 4). The strongest correlation was observed between DLQI $\mathrm{I}_{\text {baseline }}$ and $\mathrm{PASI}_{3 \text { months }}$, as well as

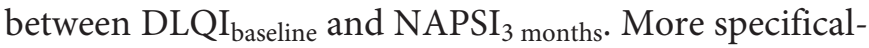
ly, the DLQI baseline $-\mathrm{NAPSI}_{3 \text { months }}$ values demonstrated the highest correlation coefficient $(r=0.515, p<0.001$, parametric analysis). Additionally, a moderate correlation was found between $\mathrm{PASI}_{\text {baseline }}$ and $\mathrm{DLQI}_{3 \text { months. }}$ Correla- 
tions between the remaining paired-indexes were weak or weak-to-moderate either on parametric or on nonparametric analysis.

\section{Discussion}

In the era of biologic drugs and new molecules, evaluation of the response to treatment using evidence-based tools is indispensable. Although the plaque type and nail psoriasis often coexist, the statistical correlation between indexes measuring the severity of these phenotypes, as well as their impact on the quality of life, has not been completely understood, as yet. Furthermore, there is a paucity of data regarding the short-term relationship of these indexes.

In the literature, there are several studies investigating the correlation between PASI, NAPSI, and DLQI. These indexes are usually studied in pairs, either at baseline or at various time points during treatment. The results of these studies are rather controversial, as some document strong correlations, whereas others find weak or no correlation at all [7-18].

Although the psychological burden of nail psoriasis has been emphasized in recently published articles $[6,19]$, there are few data concerning the NAPSI-DLQI relationship at baseline. Kyriakou et al. [7] explored this correlation in 54 patients with chronic plaque psoriasis and nail involvement who were not in any treatment at the time of their evaluation. The authors concluded that there was a strong positive correlation between DLQI and NAPSI $(r=0.56, p=0.016)$. This finding coincides with the one in our study $(r=0.579, p<0.001)$.

As far as the relationship between NAPSI and PASI at baseline is concerned, Hallaji et al. [8] found a statistically significant weak-to-moderate correlation (Pearson's correlation coefficient $=0.33, p<0.0001$ ) between NAPSI and PASI. In contrast, Rich et al. [9] found no correlation between these 2 indexes. In our study, we found a weakto-moderate correlation, in accordance with the study of Hallaji et al. [8]. Even though nail psoriasis is often associated with a greater extent of skin disease [10], the weakto-moderate correlation can be attributed to the fact that some patients present with a milder skin disease (PASI < 10) that coexists with a more severe nail involvement and vice versa.

Regarding the correlation between PASI and DLQI at baseline, this has been shown in the past to be positive but weak [11]. The strong correlation observed in our study may be due to the fact that nail involvement, which was
Table 4. Statistical correlation of indexes from baseline to 3 months

\begin{tabular}{|c|c|c|c|c|}
\hline & \multicolumn{2}{|c|}{$\mathrm{DLQI}_{3 \text { months }}$} & \multicolumn{2}{|c|}{$\mathrm{NAPSI}_{3 \text { months }}$} \\
\hline & $r$ & $p$ & $r$ & $p$ \\
\hline \multicolumn{5}{|l|}{ PASI $_{\text {baseline }}$} \\
\hline Pearson & 0.338 & 0.033 & 0.147 & 0.367 \\
\hline \multirow[t]{3}{*}{ Spearman } & 0.294 & 0.066 & 0.219 & 0.174 \\
\hline & \multicolumn{2}{|c|}{$\mathrm{PASI}_{3 \text { months }}$} & \multicolumn{2}{|c|}{$\mathrm{NAPSI}_{3 \text { months }}$} \\
\hline & $r$ & $p$ & $r$ & $p$ \\
\hline
\end{tabular}

\begin{tabular}{|c|c|c|c|c|}
\hline \multicolumn{5}{|l|}{ DLQI $_{\text {baseline }}$} \\
\hline Pearson & 0.433 & 0.005 & 0.515 & 0.001 \\
\hline \multirow[t]{3}{*}{ Spearman } & 0.465 & 0.003 & 0.453 & 0.003 \\
\hline & \multicolumn{2}{|c|}{$\mathrm{PASI}_{3 \text { months }}$} & \multicolumn{2}{|c|}{$\mathrm{DLQI}_{3 \text { months }}$} \\
\hline & $r$ & $p$ & $r$ & $p$ \\
\hline \multicolumn{5}{|l|}{ NAPSI $_{\text {baseline }}$} \\
\hline Pearson & 0.190 & 0.241 & 0.267 & 0.096 \\
\hline Spearman & 0.266 & 0.097 & 0.318 & 0.046 \\
\hline
\end{tabular}

invariably present among our patients, contributes significantly to quality of life impairment and is frequently associated with higher DLQI scores [6]. Most studies investigating the PASI-DLQI statistical relationship at baseline have recruited patients suffering from plaque psoriasis, regardless of the presence of nail involvement $[11,12]$.

Studies on indexes correlation at different time points after treatment initiation have been mainly focused on the PASI-DLQI relationship. Mattei et al. [13] reviewed the short-term (10-16 weeks) correlation of these indexes in patients with chronic, moderate-to-severe plaque psoriasis under biologic therapy. They concluded that the reduction in PASI correlates with changes in DLQI from baseline through weeks $10-16\left(r^{2}=\right.$ 0.80 ). In a more recent study [14] of 120 patients with plaque type psoriasis treated with ustekinumab, a correlation value of $0.57(p<0.001)$ between PASI and DLQI in the period from baseline to 4 months was observed.

A literature review revealed previous studies investigating correlations between indexes among patients under treatment with agents of different therapeutic categories, as in our study. Silva et al. [15] found no correlation between PASI and DLQI before and after 2 months of treatment in 35 patients who received either oral methotrexate or acitretin. They attributed their findings to in- 
creased disease acceptance and lower socioeconomic parameters. In contrast, Schäfer et al. [12] studied 93 patients who received various treatment regimens, and they found a strong correlation $(r=0.5)$ between the average improvement in PASI and DLQI 42.5 days after treatment initiation. This is in agreement with our observation that a strong correlation exists between these indexes at 3 months.

It must be noted that the statistical correlation between NAPSI and PASI or NAPSI and DLQI, at different time points, has been rarely studied. Rich et al. [9] observed a weak correlation (Spearman's correlation $=0.116, p=$ 0.078 ) between the percent improvement in PASI and NAPSI scores at week 10 in patients with skin and nail psoriasis treated with infliximab. We found a correlation coefficient of $0.215(p=0.102)$ in alignment with the authors' argument that a slower improvement in nail disease is anticipated compared to the skin disease response. Concerning the NAPSI-DLQI correlation after treatment, several studies have shown that systemic treatment results in significant improvement in both NAPSI and DLQI or other health-related quality of life assessment tools [16-18]. In our study, we found a strong correlation between NAPSI and DLQI at 3 months. This finding highlights the fact that although skin disease may significantly improve with systemic therapy the slower response of nail disease to treatment continues to exert a negative impact on the quality of life.

Lastly, in an attempt to identify a baseline index that could predict the response at 3 months, we performed an analysis of the period from baseline to 3 months. Interestingly, DLQI baseline was strongly correlated to NAP$\mathrm{SI}_{3}$ months and $\mathrm{PASI}_{3}$ months. The strongest correlation was observed between DLQI baseline and $\mathrm{NAPSI}_{3}$ months $(r=$ $0.515, p<0.001$, parametric analysis). This can be interpreted as follows: patients with moderate-to-severe plaque type psoriasis coexisting with nail disease, who presented with a higher impact on their quality of life (higher DLQI score) during their initial evaluation, are expected to exhibit an inferior response to systemic therapy in the short term. It appears that nail disease responds less well to treatment than skin disease. This finding also provides further evidence of the negative impact that nail psoriasis has on the quality of life. Although many consider DLQI to be a subjective index with some drawbacks, it remains the most frequently used. This explains the fact that regulatory bodies have embodied patient-reported outcomes in their recommendations when it comes to the conduction of clinical trials [20].
Table 5. Take-home messages

A clinician should always look for clues of nail disease in a psoriatic patient.

Nail involvement is strongly associated with the psychological burden and can independently affect the therapeutic response.

Future trials concerning patients with skin and nail psoriasis should always include a quality of life assessment tool together with clinical severity indexes.

NAPSI, PASI and DLQI are reliable, reproducible, and positively correlated indexes and are, therefore, suggested for the evaluation of patients suffering from skin and nail psoriasis.

The limitations of this study include its small sample size and the absence of a control group. Although it is more complicated to interpret, the enrollment of patients under various systemic therapies rather than a specific therapeutic option reflects more effectively real-life therapeutic practices for nail psoriasis. In addition, we preferred to evaluate only systemic and not topical treatments, as a more rapid and efficient response ( $90 \%$ reduction in PASI or more) is often anticipated with modern systemic therapies [21]. Moreover, this was a prospective study using already validated indexes that were assessed in a real-life setting and not during a therapeutic protocol. To our knowledge, this is the first study to explore the statistical correlation between NAPSI, PASI, and DLQI simultaneously before and after the administration of systemic therapy for skin and nail psoriasis. We were able to identify a baseline index that may act as a short-term predictor of the response to treatment. Our results may prove to be useful in future clinical trials, as well as in everyday clinical practice (Table 5).

In conclusion, the present study brings into the spotlight nail psoriasis and its impact on the quality of life. The aforementioned correlations indicate that nail disease is strongly associated with the psychological burden and, consequently, it should always be sought by the clinician as it can independently affect the therapeutic outcome. Moreover, the observed positive correlations between NAPSI, PASI, and DLQI further support their simultaneous utilization within the context of clinical trials, as well as in everyday practice. Even though various severity evaluation instruments have been claiming their position, clinicians should keep in mind that these 3 widelyused tools will provide them with robust scientific data when assessing the treatment response in patients suffering from skin and nail psoriasis. 


\section{Statement of Ethics}

All of the patients were informed about the methods and aim of this study and gave their consent. The study was approved by the Ethics Committees of both hospitals.

\section{Disclosure Statement}

The authors declare no conflict of interests.

\section{References}

1 Naldi L, Svensson A, Diepgen T, Elsner P, Grob JJ, Coenraads PJ, et al.; European Dermato-Epidemiology Network. Randomized clinical trials for psoriasis 1977-2000: the EDEN survey. J Invest Dermatol. 2003 May;120(5):738-41.

2 Paul C, Gourraud PA, Bronsard V, Prey S, Puzenat E, Aractingi S, et al. Evidence-based recommendations to assess psoriasis severity: systematic literature review and expert opinion of a panel of dermatologists. J Eur Acad Dermatol Venereol. 2010 Apr;24 Suppl 2:2-9.

3 de Vries AC, Bogaards NA, Hooft L, Velema $M$, Pasch M, Lebwohl M, et al. Interventions for nail psoriasis. Cochrane Database Syst Rev. 2013 Jan;(1):CD007633.

4 Finlay AY, Khan GK. Dermatology Life Quality Index (DLQI) - a simple practical measure for routine clinical use. Clin Exp Dermatol. 1994 May;19(3):210-6.

5 de Berker DA, Baran R, Dawber RP. The nail in dermatological diseases. In: Baran R, Dawber RP, de Berker DA, Haneke E, Tosti A, editors. Baran and Dawber's diseases of the nails and their management. 3rd ed. Malden: Blackwell Science; 2001. pp. 172-222.

6 Baran R. The burden of nail psoriasis: an introduction. Dermatology. 2010;221 Suppl 1: $1-5$.

7 Kyriakou A, Patsatsi A, Sotiriadis D. The agreement among the different ways of measuring NAPSI and their correlation with DLQI. J Dtsch Dermatol Ges. 2014 Nov;12(11):1051-3.

8 Hallaji Z, Badaeijndaghi F, Akbarzadeh M, Seyedi SZ, Barzegari M, Noormohammadpour $\mathrm{P}$, et al. A significant association exists between the severity of nail and skin involvement in psoriasis J Am Acad Dermatol. 2012 Jan;66(1):12-3.
9 Rich P, Griffiths CE, Reich K, Nestle FO, Scher RK, Li S, et al. Baseline nail disease in patients with moderate to severe psoriasis and response to treatment with infliximab during 1 year. J Am Acad Dermatol. 2008 Feb;58(2): 224-31.

10 de Jong EM, Seegers BA, Gulinck MK, Boezeman JB, van de Kerkhof PC. Psoriasis of the nails associated with disability in a large number of patients: results of a recent interview with 1,728 patients. Dermatology. 1996; 193(4):300-3.

11 Augustin M, Krüger K, Radtke MA, Schwippl I, Reich K. Disease severity, quality of life and health care in plaque-type psoriasis: a multicenter cross-sectional study in Germany. Dermatology. 2008;216(4):366-72.

12 Schäfer I, Hacker J, Rustenbach SJ, Radtke M, Franzke N, Augustin M. Concordance of the Psoriasis Area and Severity Index (PASI) and patient-reported outcomes in psoriasis treatment. Eur J Dermatol. 2010 Jan-Feb;20(1): 62-7.

13 Mattei PL, Corey KC, Kimball AB. Psoriasis Area Severity Index (PASI) and the Dermatology Life Quality Index (DLQI): the correlation between disease severity and psychological burden in patients treated with biological therapies. J Eur Acad Dermatol Venereol. 2014 Mar;28(3):333-7.

14 Hesselvig JH, Egeberg A, Loft ND, Zachariae C, Kofoed K, et al. Correlation between dermatology life quality index and psoriasis area and severity index in patients with psoriasis treated with ustekinumab. Acta Derm Venereol. 2018 Mar;98(3):335-9.
15 Silva MF, Fortes MR, Miot LD, Marques SA Psoriasis: correlation between severity index (PASI) and quality of life index (DLQI) in patients assessed before and after systemic treatment. An Bras Dermatol. 2013 Sep-Oct;88(5): 760-3.

16 Rigopoulos D, Gregoriou S, Stratigos A, Larios G, Korfitis C, Papaioannou D, et al. Evaluation of the efficacy and safety of infliximab on psoriatic nails: an unblinded, nonrandomized, open-label study. Br J Dermatol. 2008 Aug;159(2):453-6.

17 Rigopoulos D, Gregoriou S, Lazaridou E, Belyayeva E, Apalla Z, Makris M, et al. Treatment of nail psoriasis with adalimumab: an open label unblinded study. J Eur Acad Dermatol Venereol. 2010 May;24(5):530-4.

18 Reich K, Gooderham M, Bewley A, Green L, Soung J, Petric R, et al. Safety and efficacy of apremilast through 104 weeks in patients with moderate to severe psoriasis who continued on apremilast or switched from etanercept treatment: findings from the LIBERATE study. J Eur Acad Dermatol Venereol. 2018 Mar;32(3):397-402.

19 Augustin M, Reich K, Blome C, Schäfer I, Laass A, Radtke MA. Nail psoriasis in Germany: epidemiology and burden of disease. Br J Dermatol. 2010 Sep;163(3):580-5.

20 Langenbruch A, Radtke MA, Gutknecht M, Augustin M. Does the dermatology life quality index (DLQI) underestimate the disease- specific burden of psoriasis patients? J Eur Acad Dermatol Venereol. 2018 Aug;33(1):123-7.

21 Kim HJ, Lebwohl MG. Biologics and Psoriasis: The Beat Goes On [Epub 2018 Nov1]. Dermatol Clin. 2019 Jan;37(1):29-36. 